\title{
Endoscopic Ultrasonography Can Prevent Unnecessary Diagnostic Endoscopic Retrograde Cholangiopancreatography Even in Patients with High Likelihood of Choledocholithiasis and Inconclusive Ultrasonography: Results of a Prospective Study
}

\author{
Ruchir Patel, Meghraj Ingle, Dhaval Choksi, Prateik Poddar, Vikas Pandey and Prabha Sawant \\ Department of Gastroenterology, Lokmanya Tilak Municipal Medical College and Hospital, Mumbai, India
}

Background/Aims: Endoscopic retrograde cholangiopancreatography (ERCP) is the initial therapy recommended for patients with high likelihood of choledocholithiasis. To determine whether endoscopic ultrasonography (EUS) can prevent diagnostic ERCPs in patients with high probability of choledocholithiasis and inconclusive ultrasonography (US).

Methods: All patients with high likelihood of choledocholithiasis and negative US underwent EUS. ERCP was performed for the patients who showed a definite stone/sludge on EUS. Patients without choledocholithiasis were followed up for 3 months. The primary outcome was avoidance of diagnostic ERCP.

Results: We included 78 patients (51 women; 27 men). Of these, 25 and 7 (total 41\%) were diagnosed with choledocholithiasis and sludge, respectively; stone/sludge was removed in $96.9 \%$ of the patients. EUS ruled out choledocholithiasis in 38 patients (48.7\%). Two of them were found to have choledocholithiasis on follow-up. The sensitivity, specificity, positive and negative predictive value of EUS for detecting choledocholithiasis were $93.9 \%, 97.3 \%, 96.9 \%$, and $94.7 \%$, respectively. Unnecessary ERCP was avoided in $57.7 \%$ of the patients by using the EUS-first approach.

Conclusions: EUS is a highly accurate and safe procedure. EUS can replace ERCP as the initial investigation in patients with a high probability of choledocholithiasis. It avoids unnecessary ERCP; hence, decreasing related costs and complications. Clin Endosc 2017;50:592-597

Key Words: Endosonography; High likelihood; Choledocholithiasis; Unnecessary endoscopic retrograde cholangiopancreatography; Inconclusive imaging

\section{INTRODUCTION}

Choledocholithiasis can occur in 3\%-16\% of patients with gallbladder stones. ${ }^{1,2}$ It can be present in $10 \%-20 \%$ of patients undergoing cholecystectomy and in $18 \%-21 \%$ of those with

Received: December 26, 2016 Revised: March 18, 2017

Accepted: May 11, 2017

Correspondence: Ruchir Patel

Department of Gastroenterology, Lokmanya Tilak Municipal Medical College and Hospital, 1st Floor, College Building, Sion, Mumbai 400022, India

Tel: +91-75-0606-5561, Fax: +91-22-2406-3088, E-mail: ruchir.cn@gmail.com

(cc) This is an Open Access article distributed under the terms of the Creative Commons Attribution Non-Commercial License (http://creativecommons.org/ licenses/by-nc/3.0) which permits unrestricted non-commercial use, distribution, and reproduction in any medium, provided the original work is properly cited. gallstone pancreatitis. ${ }^{3}$ Many studies over the past decade have shown that endoscopic ultrasonography (EUS) is highly sensitive and its accuracy is comparable with endoscopic retrograde cholangiopancreatography (ERCP) in detecting common bile duct (CBD) stones. ${ }^{4.7}$ EUS has been shown to be more sensitive and specific than other noninvasive modalities such as abdominal ultrasonography (US), abdominal computed tomography (CT), and magnetic resonance cholangiopancreatography (MRCP). ${ }^{8,9}$ Thereby, it is postulated that EUS can prevent unnecessary diagnostic ERCP and its complications in patients with suspected CBD stone.

The recent American Society for Gastrointestinal Endoscopy (ASGE) practice guideline suggests a stratified approach to investigate suspected choledocholithiasis based on 
the three likelihood categories: high, intermediate, and low. ${ }^{10}$ An ample number of studies showed EUS to be the preferred modality in patients with an intermediate probability of having a CBD stone. ${ }^{11-13}$ However, for the high-probability patients, studies that directly compared EUS with ERCP are rare. Awaiting the data, ERCP remains the recommended initial therapy for the high-probability group. ${ }^{10}$

Therefore, the aim of our study was to assess whether EUS can avoid unnecessary diagnostic ERCPs in patients with a high likelihood of choledocholithiasis and negative imaging.

\section{MATERIALS AND METHODS}

\section{Study design}

This was a single-center prospective study conducted in the department of gastroenterology at a large tertiary care referral center in Western India. The study was approved by the institutional ethics committee and followed the ethical

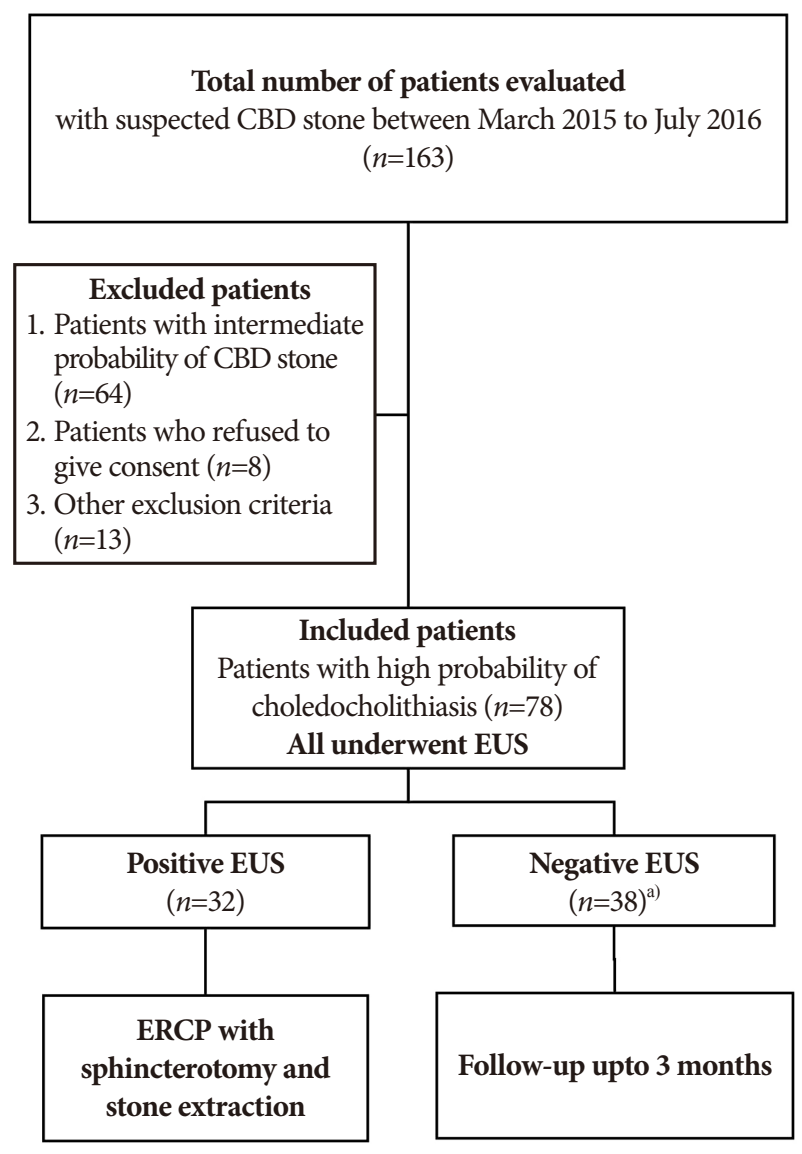

Fig. 1. Study protocol with details of the inclusion and exclusion criteria. $\mathrm{CBD}$, common bile duct; ERCP, endoscopic retrograde cholangiopancreatography. ${ }^{a)}$ Eight patients had alternative diagnosis on endoscopic ultrasonography (EUS). guidelines of the Declaration of Helsinki. Our hospital has a large referral gastrointestinal endoscopy unit that handles approximately 500 EUS and 400 ERCPs annually. All the patients admitted under the suspicion of choledocholithiasis or referred for EUS for the same indication between March 2015 and July 2016 were screened. Patients who fulfilled the inclusion criteria were enrolled in the study cohort. Written informed consent was sought from all the patients. Data on demographic characteristics; clinical features; blood biochemical tests, including liver function tests (LFT); and US findings were collected. All the patients underwent EUS first. EUS features consistent with stone or sludge were termed as a positive EUS result. ERCP with sphincterotomy and stone extraction were performed for patients with positive EUS results. Those with a negative EUS result were followed up for at least 3 months by clinical evaluation every 15 days, LFT weekly until normalization, and repeat US if required. ERCP and stone removal were performed if a CBD stone was detected during follow-up. EUS findings other than choledocholithiasis were also noted and managed in accordance with the diagnosis. The study protocol and enrolment of the patients are shown in Fig. 1.

\section{Patient selection}

The patients who presented with symptoms of biliary colic were included in the study if they had inconclusive imaging and any one of the following ASGE criteria suggestive of high clinical likelihood of choledocholithiasis: ${ }^{10}$ symptoms consistent with cholangitis, bilirubin level of $>4 \mathrm{mg} / \mathrm{dL}$, and dilated CBD on US ( $\geq 6 \mathrm{~mm} ; \geq 10 \mathrm{~mm}$ if post-cholecystectomy status), with a bilirubin level of $1.8-4 \mathrm{mg} / \mathrm{dL}$ or suspicious $\mathrm{CBD}$ stone on imaging. Inconclusive imaging meant abdominal US that failed to show the cause of biliary obstruction. CT or MRCP was not mandatory for the inclusion of patients. The exclusion criteria for the study were patients aged $<18$ years, pregnancy, previously diagnosed biliary pathology other than choledocholithiasis, patients with a low performance status who were considered high-risk for sedation by an anesthetist, and those not willing to provide informed consent.

\section{Procedures}

All EUS procedures were performed by a single expert endoscopist (MI) with $>3$ years of experience in the field of diagnostic and therapeutic EUS. The procedures were carried out by using a linear echoendoscope (GF-UCT180; Olympus Medical, Tokyo, Japan) under conscious sedation with intravenous midazolam and pentazocine. EUS was performed from the gastroesophageal junction, antrum, and first and second parts of the duodenum for complete $\mathrm{CBD}$ evaluation. 
CBD stone was characterized by echogenic focus in the bile duct with acoustic shadowing, while sludge manifests as a layering echogenic material without shadows. Therapeutic ERCP was performed by using a side-viewing ERCP scope (TJF-150; Olympus Medical, Tokyo, Japan) by the same endoscopist under the same sedation in case of positive EUS findings. The procedure steps included bile duct access with guide wire by using a biliary sphincterotome, contrast injection in CBD to locate the stone or sludge, biliary sphincterotomy, and stone or sludge extraction by using a stone extraction balloon catheter followed by 7- or 10-F biliary plastic stent placement. EUS and ERCPs were performed by a single experienced endoscopist to minimize variation in procedure results as both ERCP and especially EUS are highly operator dependent.

\section{Study outcomes}

The primary outcome of the study was avoidance of unnecessary diagnostic ERCP. The sensitivity, specificity, negative predictive value (NPV), and positive predictive value (PPV) of EUS for detecting CBD stones were considered secondary outcomes. The laboratory and imaging parameters proposed in the high likelihood criteria were compared between the patients with and those without choledocholithiasis on EUS to validate their predictive accuracy. These results, along with the safety of EUS and ERCP procedures, were the additional outcomes of the study.

\section{Statistical analysis}

Descriptive data were assessed as mean and standard deviation (SD). The sensitivity, specificity, NPV, and PPV of EUS for detection of CBD stones were calculated by using the ERCP findings as the gold standard. Factors associated with $\mathrm{CBD}$ stones were assessed by using univariate analysis. Chisquare test or Fisher exact test, when the expected number of cells was $<5$, were applied to analyze categorical variables. On the other hand, non-parametric continuous variables were analyzed by using the Mann-Whitney $U$ test; and parametric continuous data, by unpaired $t$ test. A $p$-value of $<0.05$ was considered statistically significant. All statistical analyses were performed by using the SPSS version 17.0 software (SPSS Inc., Chicago, IL, USA).

\section{RESULTS}

\section{Study cohort}

The study cohort included 78 patients aged $45.5 \pm 15.1$ years (mean \pm SD). Most of the patients were female $(n=51,66 \%)$. The numbers of patients who fulfilled the criteria of high
Table 1. Patient Distribution according to Various ASGE Criteria for High Probability of Choledocholithiasis

\begin{tabular}{lcc}
\hline & $\begin{array}{c}\text { Number of } \\
\text { patients }(\boldsymbol{n})\end{array}$ & $\begin{array}{c}\text { Percentage } \\
(\%)\end{array}$ \\
\hline $\begin{array}{l}\text { Patients with high likelihood of } \\
\text { CBD stone }\end{array}$ & 78 & 100 \\
Dilated CBD with altered LFT & 56 & 71.8 \\
Bilirubin $>4$ mg/dL & 19 & 24.4 \\
Clinical cholangitis & 3 & 3.8 \\
\hline
\end{tabular}

ASGE, American Society for Gastrointestinal Endoscopy; CBD, common bile duct; LFT, liver function tests.

Table 2. Clinical and Laboratory Characteristics of the Study Cohort

\begin{tabular}{lc}
\hline Patient characteristics & Total patients $(\boldsymbol{n = 7 8 )}$ \\
\hline Male $(n, \%)$ & $27(34)$ \\
Female $(n, \%)$ & $51(66)$ \\
Age $($ mean \pm SD) $(\mathrm{yr})$ & $45.5 \pm 15.1$ \\
White cells $\left(/ \mathrm{mm}^{3}\right)($ mean $)$ & 8,500 \\
LFT $($ mean \pm SD $)$ & \\
Bilirubin $(\mathrm{mg} / \mathrm{dL})$ & $3.4 \pm 3.8$ \\
ALT $(\mathrm{U} / \mathrm{L})$ & $167 \pm 130$ \\
AST $(\mathrm{U} / \mathrm{L})$ & $138 \pm 126$ \\
ALP $(\mathrm{U} / \mathrm{L})$ & $368 \pm 256$ \\
Dilated CBD $(n, \%)$ & $56(71.8 \%)$ \\
\hline
\end{tabular}

$\mathrm{SD}$, standard deviation; LFT, liver function tests; ALT, alanine aminotransferase; AST, aspartate aminotransferase; ALP, alkaline phosphatase; CBD, common bile duct.

likelihood of choledocholithiasis are shown in Table 1. All the patients had inconclusive US results. Among them, 56 (71.8\%) had a dilated CBD. Bilirubin level was the most commonly deranged LFT component (95\%). Detailed demographic and laboratory characteristics of the study population are shown in Table 2.

\section{Primary and secondary outcomes}

EUS detected choledocholithiasis in 25 patients (32.1\%); and CBD sludge, in 7 patients (8.9\%). The mean stone size was $8 \mathrm{~mm}$ (range, 4-15 mm). Subsequent ERCP extracted stone or sludge in 31 of the 32 patients (96.9\%). EUS was used to rule out choledocholithiasis in 38 patients (48.7\%). They were discharged and followed up for a minimum of 3 months, with a mean follow-up period of 4.2 months. In these patients, CBD stones were diagnosed during follow-up imaging in 2 patients who underwent ERCP, on follow-up day 10 in 1 patient, and on day 14 in 1 patient. LFT results were persistently elevated, and recurrent biliary colic appeared in both patients. The rest of the patients were symptom-free. The sensitivity and specificity of EUS for detecting CBD stone were $93.9 \%$ and $97.3 \%$, 
respectively. EUS had a high predictive accuracy for detecting and ruling out stones in suspected choledocholithiasis, with a PPV of $96.9 \%$ and NPV of $94.7 \%$. Diagnostic ERCPs were avoided in 45 patients (57.7\%). Various EUS results are shown in Table 3.

Table 3. EUS Findings of the Study

\begin{tabular}{lcc}
\hline EUS findings & $\begin{array}{c}\text { Number of } \\
\text { patients }(\boldsymbol{n})\end{array}$ & $\begin{array}{c}\text { Percentage } \\
(\mathbf{\%})\end{array}$ \\
\hline CBD stone & 25 & 32.1 \\
CBD sludge & 7 & 8.9 \\
Negative EUS & 38 & 48.7 \\
Others & 8 & 10.3 \\
Cholangiocarcinoma & 2 & 2.6 \\
Ampullary Carcinoma & 1 & 1.3 \\
Carcinoma GB & 1 & 1.3 \\
Extrinsic compression & 2 & 2.6 \\
Round worm in CBD & 1 & 1.3 \\
Mirrizi's syndrome & 1 & 1.3 \\
\hline
\end{tabular}

EUS, endoscopic ultrasonography; CBD, common bile duct; GB, gall bladder.

Table 4. Univariate Analysis of the Factors Potentially Associated with Presence of Choledocholithiasis. Patients Who were Diagnosed with Other Etiologies on EUS $(n=8)$ were Excluded from the Univariate Analysis

\begin{tabular}{lccl}
\hline & $\begin{array}{c}\text { Stone present } \\
(\boldsymbol{n}=\mathbf{3 2})\end{array}$ & $\begin{array}{c}\text { Stone absent } \\
(\boldsymbol{n}=\mathbf{3 8})\end{array}$ & $\boldsymbol{p}$-value \\
\hline LFT (mean \pm SD) & & & \\
Bilirubin (mg/dL) & $3.8(3.4)$ & $3(3.2)$ & 0.3 \\
ALT (U/L) & $185(161)$ & $159(119)$ & 0.4 \\
AST (U/L) & $153(123)$ & $137(132)$ & 0.6 \\
ALP (U/L) & $397(272)$ & $362(248)$ & 0.57 \\
Dilated CBD $(n, \%)$ & $24(75 \%)$ & $26(69 \%)$ & 0.6 \\
\hline
\end{tabular}

EUS, endoscopic ultrasonography; LFT, liver function tests; SD, standard deviation; ALT, alanine aminotransferase; AST, aspartate aminotransferase; ALP, alkaline phosphatase; CBD, common bile duct.

Table 5. Adverse Events Seen with EUS and ERCP Procedures during the Study

\begin{tabular}{lcc}
\hline Adverse event & EUS $(\boldsymbol{n}=\mathbf{7 8})$ & ERCP $(\boldsymbol{n}=\mathbf{3 3})$ \\
\hline Pancreatitis & 0 & 2 \\
Bleeding & 1 & 5 \\
Hypoxia & 1 & 3 \\
Perforation & 0 & 0 \\
Cholangitis & 0 & 0 \\
Mortality & 0 & 0 \\
Sedation related complications & 0 & 1 \\
\hline
\end{tabular}

EUS, endoscopic ultrasonography; ERCP, endoscopic retrograde cholangiopancreatography.

\section{Additional outcomes}

The univariate analysis of the factors potentially associated with choledocholithiasis is shown in Table 4. Neither the laboratory parameters such as bilirubin $(p=0.3)$, alanine aminotransferase (ALT; $p=0.4$ ), aspartate aminotransferase (AST; $p=0.6$ ), and alkaline phosphatase (ALP) levels $(p=0.57)$ nor the imaging parameters such as dilated CBD on US $(p=0.6)$ were significantly associated with the presence of choledocholithiasis.

\section{Other etiologies}

Apart from CBD stones, EUS also detected various other etiologies in 8 patients (10.3\%). On EUS, malignant biliary obstruction was identified in 4 patients (5.2\%). One of them had an ampullary carcinoma, 2 had a distal cholangiocarcinoma, and 1 had a carcinoma of the gallbladder neck. Two patients (2.6\%) had extrinsic compression on CBD, 1 (1.3\%) had roundworm infestation, and 1 (1.3\%) had Mirizzi's syndrome. None of the 8 patients had choledocholithiasis. Roundworm on EUS was detected as a hypoechoic linear structure with hyperechoic walls within the CBD without any acoustic shadowing. Roundworm was removed from the CBD by ERCP and balloon sweeps. The rest of the cases were managed in accordance with the diagnosis. Operable malignancies were referred for surgical exploration, and inoperable ones were treated accordingly.

\section{Adverse events}

No serious adverse events occurred during either the EUS or ERCP procedures. Although no head-to-head comparison was performed between EUS and ERCP, complications were less with EUS than with ERCP. All the adverse events were managed conservatively. The adverse events that occurred during the study are described in Table 5.

\section{DISCUSSION}

Several studies have invalidated the use of biochemical and imaging parameters to predict choledocholithiasis. ${ }^{14,15}$ EUS is sensitive and highly accurate for diagnosing choledocholithiasis as compared with MRCP and ERCP, with up to $94 \%$ sensitivity and $95 \%$ specificity. ${ }^{8.9}$ EUS remains highly sensitive for stones $<5 \mathrm{~mm}$, where the sensitivity of MRCP has been reported to be as low as 33\%-71\%. ${ }^{16,17}$ Current guidelines still rely on a risk stratification approach for $\mathrm{CBD}$ stone based on various prognostic parameters. They recommend pre-procedure EUS or MRCP for patients with intermediate probability of choledocholithiasis and direct ERCP for the high-probability group. ${ }^{10}$ Considering the relatively 
high rates of complications and invasive nature of ERCP, EUS has been proposed even in the high likelihood group to avoid unnecessary diagnostic ERCP.

The present study assessed the role of EUS for detection of CBD stone in the high-probability group. Only $41 \%$ of the patients categorized as high-risk according to the ASGE guideline actually had choledocholithiasis. This confirms the unreliability of the prognostic models in the high-probability group. Almost similar results were obtained in a study by Prachayakul et al. (38.68\%). ${ }^{11}$ Several other studies also reported a stone detection rate of only up to $60 \%$ in high likelihood patients. ${ }^{18-20}$ Unnecessary diagnostic ERCP was avoided in $57.7 \%$ of the patients by using the "EUS first" approach in the present study.

The sensitivity and specificity of EUS in detecting CBD stones in our study were $93.9 \%$ and $97.3 \%$, respectively. Only 1 EUS showed a false-positive result, and 2 showed false-negative results. False-negative results were possibly attributed to a small size of stones. Prachayakul's group showed $100 \%$ sensitivity and $80 \%$ specificity; however, it included both high- and intermediate-probability patients. ${ }^{11}$ Another study showed a sensitivity and specificity of $91 \%$ and $100 \%$, respectively, in the high-risk cohort. ${ }^{19}$ The predictive accuracy of EUS in the present study was almost similar to that of others. The marginally lower sensitivity and predictive accuracy can be attributed to the relatively recent availability of EUS in our center and shorter experience of the endoscopists in performing EUS. Our study results were analogous to those of studies performed in larger EUS centers.

No significant association was found between any of the clinical, laboratory, or imaging parameters proposed in the high likelihood criteria and for choledocholithiasis in the present study. A randomized controlled trial (RCT) by Lee et al. found similar results. ${ }^{18}$ However, one study showed an association of elevated ALP levels and positive results of EUS performed within 7 days of symptom onset. ${ }^{11} \mathrm{~A}$ study from Italy showed only CBD diameter to be the independent predictor of choledocholithiasis. ${ }^{13}$ The discordant results from multiple studies show the unreliability of the commonly used biochemical and radiological predictors of the presence of CBD stone even for high-probability groups. Therefore, we suggest that EUS should be performed in all cases of highly probable choledocholithiasis.

Few studies have also shown significant cost benefits of the "EUS first" approach in comparison with MRCP before ERCP or ERCP alone in patients with low to intermediate likelihood of choledocholithiasis. ${ }^{21}$ The same approach can be applied to high likelihood patients in spite of the higher need for ERCP in this cohort. Although the present study did not perform a cost analysis, the "EUS first" approach might be less costly for high likelihood groups, considering the extremely high costs of unnecessary ERCP, hospital stay, unnecessary investigations, and medications. However, further studies are required to assess the cost-effectiveness in this cohort.

EUS detected various etiologies of biliary obstruction other than choledocholithiasis in $10.3 \%$ of cases. The high rate of alternative diagnosis can be ascribed to an inappropriate clinical history given by few patients who were referred for EUS in an outpatient basis. Those patients were managed in accordance with their diagnosis. Nevertheless, this shows the usefulness of EUS in the detection of other biliary diseases that might not be found on ERCP. All EUS procedures were performed without any complications.

This stud had a few limitations. First, it was not an RCT. Head-to-head comparison between EUS and ERCP was not performed, and the diagnostic accuracy of EUS was calculated and compared with that of ERCP as the gold standard. ERCP can yield false results, as it is operator and technology dependent. Second, the minimum follow-up period for patients with negative EUS results was only 3 months. Longer follow-up data can produce even more accurate results. Apart from that, newly migrated stones from the gallbladder during follow-up can produce similar symptoms as missed stones. However, persistently elevated LFT results and clinical evaluation in both patient groups differentiated the two conditions in favor of missed stones.

\section{CONCLUSIONS}

The present study confirms that EUS-guided ERCP is a highly accurate and safe strategy for the management of patients with high probability of CBD stone. EUS not only identifies patients who need ERCP but also safely avoids unnecessary diagnostic ERCP, hence reducing related potential complications and significant healthcare costs. We conclude that EUS should be considered routinely instead of ERCP in all patients with a high probability of suspected choledocholithiasis. ERCP should be reserved only for patients with positive EUS results.

\section{Conflicts of Interest}

The authors have no financial conflicts of interest.

\section{Acknowledgments}

We acknowledge Dr. Shantanu Kulkarni and all the residents from the Department of Anaesthesia, Lokmanya Tilak Medical College and Hospital, for their immense support throughout the study by administering conscious sedation in the patients who were to undergo EUS and ERCP. 


\section{REFERENCES}

1. Collins C, Maguire D, Ireland A, Fitzgerald E, O'Sullivan GC. A prospective study of common bile duct calculi in patients undergoing laparoscopic cholecystectomy: natural history of choledocholithiasis revisited. Ann Surg 2004;239:28-33.

2. Shiozawa S, Tsuchiya A, Kim DH, et al. Useful predictive factors of common bile duct stones prior to laparoscopic cholecystectomy for gallstones. Hepatogastroenterology 2005;52:1662-1665.

3. Cohen ME, Slezak L, Wells CK, Andersen DK, Topazian M. Prediction of bile duct stones and complications in gallstone pancreatitis using early laboratory trends. Am J Gastroenterol 2001;96:3305-3311.

4. Polkowski M, Palucki J, Regula J, Tilszer A, Butruk E. Helical computed tomographic cholangiography versus endosonography for suspected bile duct stones: a prospective blinded study in non-jaundiced patients. Gut 1999;45:744-749.

5. Kohut M, Nowak A, Nowakowska-Dulawa E, Marek T, Kaczor R. Endosonography with linear array instead of endoscopic retrograde cholangiography as the diagnostic tool in patients with moderate suspicion of common bile duct stones. World J Gastroenterol 2003;9:612-614.

6. Buscarini E, Tansini P, Vallisa D, Zambelli A, Buscarini L. EUS for suspected choledocholithiasis: do benefits outweigh costs? A prospective, controlled study. Gastrointest Endosc 2003;57:510-518.

7. Canto MI, Chak A, Stellato T, Sivak MV Jr. Endoscopic ultrasonography versus cholangiography for the diagnosis of choledocholithiasis. Gastrointest Endosc 1998;47:439-448.

8. Tse F, Liu L, Barkun AN, Armstrong D, Moayyedi P. EUS: a meta-analysis of test performance in suspected choledocholithiasis. Gastrointest Endosc 2008;67:235-244.

9. Garrow D, Miller S, Sinha D, et al. Endoscopic ultrasound: a meta-analysis of test performance in suspected biliary obstruction. Clin Gastroenterol Hepatol 2007;5:616-623.

10. ASGE Standards of Practice Committee, Maple JT, Ben-Menachem T, et al. The role of endoscopy in the evaluation of suspected choledocholithiasis. Gastrointest Endosc 2010;71:1-9.

11. Prachayakul V, Aswakul P, Bhunthumkomol P, Deesomsak M. Diagnostic yield of endoscopic ultrasonography in patients with intermediate or high likelihood of choledocholithiasis: a retrospective study from one university-based endoscopy center. BMC Gastroenterol 2014;14:165.

12. De Lisi S, Leandro G, Buscarini E. Endoscopic ultrasonography versus endoscopic retrograde cholangiopancreatography in acute biliary pancreatitis: a systematic review. Eur J Gastroenterol Hepatol 2011;23:367374 .

13. Anderloni A, Ballarè $M$, Pagliarulo $M$, et al. Prospective evaluation of early endoscopic ultrasonography for triage in suspected choledocholithiasis: results from a large single centre series. Dig Liver Dis 2014;46:335-339.

14. Tse F, Barkun JS, Barkun AN. The elective evaluation of patients with suspected choledocholithiasis undergoing laparoscopic cholecystectomy. Gastrointest Endosc 2004;60:437-448.

15. Kim KH, Kim W, Lee HI, Sung CK. Prediction of common bile duct stones: its validation in laparoscopic cholecystectomy. Hepatogastroenterology 1997;44:1574-1579.

16. Zidi SH, Prat F, Le Guen O, et al. Use of magnetic resonance cholangiography in the diagnosis of choledocholithiasis: prospective comparison with a reference imaging method. Gut 1999;44:118-122.

17. Boraschi P, Neri E, Braccini G, et al. Choledocolithiasis: diagnostic accuracy of MR cholangiopancreatography. Three-year experience. Magn Reson Imaging 1999;17:1245-1253.

18. Lee YT, Chan FK, Leung WK, et al. Comparison of EUS and ERCP in the investigation with suspected biliary obstruction caused by choledocholithiasis: a randomized study. Gastrointest Endosc 2008;67:660-668.

19. Karakan T, Cindoruk M, Alagozlu H, Ergun M, Dumlu S, Unal S. EUS versus endoscopic retrograde cholangiography for patients with intermediate probability of bile duct stones: a prospective randomized trial. Gastrointest Endosc 2009;69:244-252.

20. Benjaminov F, Stein A, Lichtman G, Pomeranz I, Konikoff FM. Consecutive versus separate sessions of endoscopic ultrasound (EUS) and endoscopic retrograde cholangiopancreatography (ERCP) for symptomatic choledocholithiasis. Surg Endosc 2013;27:2117-2121.

21. Arguedas MR, Dupont AW, Wilcox CM. Where do ERCP, endoscopic ultrasound, magnetic resonance cholangiopancreatography, and intraoperative cholangiography fit in the management of acute biliary pancreatitis? A decision analysis model. Am J Gastroenterol 2001;96:28922899. 